\title{
Model consequences and model affect: Their effects on imitation
}

\author{
MARK H. THELEN, STEPHEN J. DOLLINGER, and MICHAEL C. ROBERTS \\ University of Missouri, Columbia, Missouri 65201 \\ and \\ T. JOHN AKAMATSU \\ Kent State University, Kent, Ohio 44240
}

\begin{abstract}
Children's imitation of a buttonpressing response was assessed as a function of model consequences and model affect. Three levels of model affect (positive, neutral, and negative) were orthogonally combined with three levels of model consequences (positive, neutral, and negative). There were no significant main or interaction effects. However, under positive model affect, subjects who observed the positive consequences model imitated significantly more than subjects who observed the no consequences model. A difference between comparable groups was not found under neutral model affect. These findings were replicated in a second experiment.
\end{abstract}

One of the most significant concepts in imitation theory and research is model consequences. A recent review by Thelen and Rennie (1972) illustrates the extent to which model consequences has received attention. It is noteworthy that, despite the substantial number of studies on model reward, relatively little attention has been given in empirical research to the model's emotional reaction following reward or punishment. A careful reading of the procedures in studies on model consequences revealed that many researchers fail to provide any reference to the model's verbal and, more importantly, nonverbal reaction following reward or punishment. However, it is likely that, even though the model reward was staged, the model showed indications of positive affect following model reward and negative affect following model'punishment (e.g., Bandura, 1965). Even if the model did not show an emotional reaction following reward or punishment, an observer still might infer that the model was experiencing a positive or negative emotional reaction.

Lerner and Weiss (1972) had children observe a model who received either a high- or a low-valued reward to which the model responded with either positive or negative affect. They found that children in the positive affect condition performed more imitative responses in playing with toys than did children in the negative affect condition. Slaby and Parke (1971)

\footnotetext{
The authors are grateful to James Moreton, Charles Bazaldua, James Holman, Sue Coverdell, and Steve McColley for their assistance with this study. The authors are also grateful to the staff at Our Lady of Lourdes and Parkade schools for their cooperation in this study. Requests for reprints should be sent to Mark $\mathbf{H}$. Thelen, Department of Psychology, McAlester Hall, University of Missouri. Columbia, Missouri 65201. Michael C. Roberts is now at Purdue University.
}

studied the influence of model consequences (positive and negative) and model affect (positive, none, or negative) in a resistance-to-deviation situation. Slaby and Parke found that model reward subjects imitated deviation behaviors more than model punishment subjects. Additionally, a significant interaction effect revealed that imitation increased in the model affect-model consequences conditions which were congruent. Surprisingly, subjects who observed a model punished after which the model expressed negative affect, showed considerable imitation. Dollinger and Thelen (1975) crossed model affect and anticipated consequences factors in a study on imitative toy play. While anticipated consequences influenced a number of play behavior measures, the main and interactive effects involving model affect were nonsignificant. In treating test-anxious college students, Jaffe and Carlson (1972) also manipulated the model's affect and consequences. This study yielded mixed results on several measures.

The results of these studies are not consistent. It appears that further research is needed to explicate the relative and interactive influence of model reward and model affect in the imitation situation. Furthermore, a socially neutral task (one which contains little likelihood of a history of reward or punishment, unlike resistance to deviation) would perhaps help to elucidate the influence of the model affect and model consequence variables.

The purpose of the present study was to investigate the separate and interactive influence of model consequences and model affect on imitation. Two experiments are reported. The second experiment, nearly identical to the first, was conducted to establish the reliability of the results from the first experiment. In each experiment, there were three levels of model 
consequences (positive, neutral, and negative) and three levels of model affect (positive, neutral, and negative).

\section{METHOD}

\begin{abstract}
Subjects
Forty-five boys and 45 girls from Grades 1,2 , and 3 of a local parochial school served as subjects in Experiment 1 . In Experiment 2, the subjects were 50 children of each sex from the same grades of a local public school. Five children of each sex were randomly assigned to each of the experimental conditions, and to a no-model control group in Experiment 2. The conditions were equated as closely as possible with respect to grade in school. The same white male undergraduate served as the experimenter for both samples, as well as on the videotapes.
\end{abstract}

\section{Experimental Task}

The task apparatus consisted of a flat rectangular box with three buttons (labeled " 1 ," " 2 ," and " 3 ") mounted on the front (Akamatsu \& Thelen, 1971). The face of a clown was painted above the buttons with white light bulbs mounted at the clown's eyes and nose. When pressed, the buttons would light up the appropriate light. A light located at the top of the clown's hat flashed at 15 -sec intervals, signaling the beginning of each trial.

The modeled responses were low-base-rate buttonpressing sequences consisting of two presses alike followed by one different. Four response patterns $(112,113,221$, and 223) comprised the critical sequences.

\section{Videotapes}

Nine videotapes were constructed, each presenting one level of the affect variable paired with one level of the consequences variable. At the beginning of each tape, the model, the rewarding agent, and the experimenter (all adult white males) were seated at a table on which the task apparatus was presented in full view. The modeling sequence began as the experimenter instructed the model to push the buttons three times in any way he liked, each time the top light came on. ${ }^{1}$ On 12 of the model's 16 trials, he pressed the buttons in one of the critical sequences described above. Thus, each critical sequence was modeled three times. In addition, two noncritical sequences (213 and 333) were each modeled twice. The four noncritical trials were intersperced among the 16 trials. The model verbalized the numbers as he pressed the buttons.

In order to equate the affect manipulations across the three levels of consequences, master tapes depicting the three affect conditions were constructed, with the consequences manipulation dubbed in on duplicate tapes. Following each critical sequence, the rewarding agent administered either positive, negative, or no consequences. Positive consequences consisted of statements indicating correct responding (e.g., "good" or "that's very good"), while negative consequences on corresponding trials consisted of negative verbalizations by the rewarder (e.g., "wrong" or "not very good"). In the no-consequence conditions, the rewarding agent was seen chewing gum-this was done on all three master tapes to allow for the dubbing-but making no statement.

Following the consequences manipulation, the model expressed positive, negative, or neutral affect on critical trials. Positive affect consisted (for exar 'ple) of a broad smile and the exclamation, "I feel good!" or a laugh followed by "hey, hey, hey!" Negative affect was expressed by a frown followed by the statements (for example) "oh yuk!" or "I feel lousy!" Selfreward and self-punishment statements were deliberately not used; only statements indicative of the model's emotional state were expressed. The neutral affect model simply directed his gaze toward the camera without making any verbalizations and without appearing pleased or displeased. Following the noncritical trials in all conditions, the model made no verbalizations, and appeared neither pleased nor displeased.

\section{Procedure}

Each subject was escorted to the experimental room individually by the experimenter and told that, after watching television, he would be able to push buttons on the same clown he would see on television. After observing one of the nine videotapes, the subject was instructed to push the buttons three times in any way he liked, each time the light came on. The subjects were given 20 trials. Experiment 2 included an additional group ( 10 subjects) who did not observe a videotape prior to pushing buttons on the clown.

\section{RESULTS AND DISCUSSION}

Each subject's imitation score was the total number of times he pressed one of the critical sequences. Table 1 presents the mean imitation scores for all conditions. A 3 (model consequences) by 3 (model affect) analysis of variance showed no significant main or interaction effects for either experiment. However, because of its particular relevance to previous research which showed model reward effects with the same task (Thelen, McGuire, Simmonds, \& Akamatsu, 1974; Thelen, Rennie, Fryrear, \& McGuire, 1972), planned comparisons were done comparing the positive-consequences and the no-consequences groups under conditions of positive model affect and under conditions of neutral model affect. Under conditions of neutral model affect, the positive- and neutral-consequences groups were not significantly different in either experiment. However, under positive model affect in Experiment 1, the positive-consequences group $(\mathrm{M}=4.4)$ imitated significantly more than the no-consequences group $(\mathrm{M}=1.5, \mathrm{p}<.05)$. This same finding was replicated in Experiment 2, wherein, with positive model affect, the positive-consequences group $(M=4.8)$ imitated significantly more than the no-consequences group $(M=2.3, p<.05)$. Because of a heterogeneity of

Table 1

Mean Imitative Responses by Condition

\begin{tabular}{|c|c|c|c|}
\hline \multirow[b]{2}{*}{ Model Consequences } & \multicolumn{3}{|c|}{ Model Affect } \\
\hline & Positive & Neutral & Negative \\
\hline
\end{tabular}

Experiment 1

Positive

No Consequences

Negative

4.4

1.7

1.5

4.2

3.1

4.1

Experiment 2

1.6

3.1

Positive

4.8

3.2

No Consequences

2.3

2.5

Negative

4.2

2.1

1.7

3.8

1.9

Note-In Experiment II the mean for no-model control subjects was.5. 
variance problem, the Mann-Whitney U statistic was also applied to these comparisons, resulting in the same conclusions. While all modeling groups in the second experiment were greater than the no-model group, only four conditions were statistically significantly greater: positive consequences-positive affect, positive consequences-neutral affect, no consequences-negative affect, and negative consequences-positive affect.

It would appear that increased imitation, as a function of model reward with a neutral task, will not occur unless the model is shown expressing some positive emotional response to the reward. In the present study, the "expressionless" face of the neutral model was in full view of the camera. A review of the videotapes of our previous research which showed increased imitation as a function of model reward (Thelen et al., 1974; Thelen et al., 1972) revealed that the model's face was not directly visible to the observer. It is possible that the observer inferred that the model was demonstrating a positive emotional response. In the studies of other researchers on model reward, it is possible that these researchers did not depict the face of the model, or that, if the frontal view of the model was provided, the model showed some positive facial-emotional expression upon being rewarded. A review of the reported procedures used in research on model reward affords little information bearing on this question. However, there is little reason to believe that the model's reaction has been deliberately controlled by researchers in investigations of model reward.

Based on the literature, it was expected that model punishment would result in less imitation than would occur in the no-consequences group. However, the low frequency of critical responses in the control group (M $=0.5$ ) disallowed the possibility of any model group emitting significantly less critical responses than the control group.

\section{REFERENCES}

Akamatsu, T. J., \& Thelen, M. H. The acquisition and performance of a socially neutral response as a function of vicarious reward. Developmental Psychology, 1971, 5, 440-445.

BANDURA. A. Influence of model's reinforcement contingencies on the acquisition of imitation responses. Journal of Personality and Social Psychology, 1965, 1, 589-595.

Dollinger, S. J., \& Thelen, M. H. Anticipated model consequences, model affect, and imitation. Developmental Psychology, 1975, 11, 390.

JAFFe, P. G., \& Carlson, P. M. Modelling therapy for test anxiety: The role of model affect and consequences. Behaviour Research and Therapy, 1972, 10, 329-339.

LERNER, L., \& WeIss, R. L. Role of value of reward and model affective response in vicarious reinforcement. Journal of Personality and Social Psychology, 1972, 21, 93-100.

SLABY, R. G., \& PARKe, R. D. Effect on resistance to deviation of observing a model's affective reaction to response consequences. Developmental Psychology, 1971, 5, 40-47.

Thelen, M. H., McGuire, D., Simmonds, D. W., \& Akamatsu, T. J. Effect of model-reward on the observer's recall of the modeled behavior. Journal of Personality and Social Psychology, 1974, 29, 140-144.

Thelen, M. H., \& Rennie, D. L. The effect of vicarious reinforcement on imitation: A review of the literature. In B. H. Maher (Ed.), Progress in experimental personality research (Vol. 6). New York: Academic Press, 1972.

Thelen, M. H., Rennie, D. L., Fryrear, J. L., \& McGuire, D. Expectancy to perform and vicarious reward: Their effects on imitation. Child Development, 1972, 42, 699-703.

\section{NOTE}

1. In Experiment 2, an additional instruction was dubbed onto the model's introduction to the task to increase the salience of the affect and consequence cues. The instruction consisted of the experimenter's statement, "There are certain good orders in which to push the buttons; some people feel good when they press the buttons in a good order."

(Received for publication July 21, 1975.) 